\title{
EPTCAST - EIN VORSCHLAG ZUR WISSENSCHAFTLICHEN VERBREITUNG IN DER BRASILIANISCHEN BERUFS- UND TECHNOLOGIEBILDUNG
}

\section{ORIGINALER ARTIKEL}

RAMOS, Rogério Luiz da Silva ${ }^{1}$, FECURY, Amanda Alves², OLIVEIRA, Euzébio de ${ }^{3}$, DENDASCK, Carla Viana ${ }^{4}$, DIAS, Claudio Alberto Gellis de Mattos ${ }^{5}$

RAMOS, Rogério Luiz da Silva. Et al. EPTCast - Ein vorschlag zur wissenschaftlichen verbreitung in der brasilianischen berufs- und technologiebildung. Revista Científica Multidisciplinar Núcleo do Conhecimento. Jahrgang 06, Ed. 06, Vol. 07, S. 89-103. Juni 2021. ISSN: 2448-0959, Zugangslink: https://www.nucleodoconhecimento.com.br/bildung-de/berufs-und-

technologiebildung, DOI: 10.32749/nucleodoconhecimento.com.br/bildung-de/berufsund-technologiebildung

\section{ZUSAMMENFASSUNG}

Diese Arbeit besteht aus einem Erfahrungsbericht über die Entwicklung von EPTCast, Bildungsprodukt während des professionellen Master-Studiengangs in Professional And Technological Education in National Network (ProfEPT) entwickelt. Basierend auf dem Mangel an wissenschaftlicher Verbreitung, die sich speziell auf den Bereich der brasilianischen beruflichen und technologischen Bildung (EPT) konzentriert, zielt diese

\footnotetext{
${ }^{1}$ Diplom im Außenhandel, Fachkraft für Produktionstechnik (UNINTER), Professor und Forscher am Institut für Grund-, Technik- und Technologiepädagogik von Amapá (IFAP), Student des Graduiertenprogramms für berufliche und technologische Bildung (PROFEPT IFAP).

${ }^{2}$ Biomedizin, PhD in Tropischen Krankheiten, Professor und Forscher des Medical Course of Macapá Campus, Federal University of Amapá (UNIFAP).

${ }^{3}$ Biologe, PhD in Tropischen Krankheiten, Professor und Forscher des Physical Education Course, Federal University ofPará (UFPA).

${ }^{4}$ Theologe, PhD in Klinischer Psychoanalyse. Er arbeitet seit 15 Jahren mit wissenschaftlicher Methodik (Forschungsmethode) in der wissenschaftlichen Produktionsberatung für Master- und Doktoranden. Spezialist für Markt- und Gesundheitsforschung, Doktorand in Kommunikation und Semiotik (PUC SP).

${ }^{5}$ Biologe, PhD in Theorie- und Verhaltensforschung, Professor und Forscher des Chemie-Studiengangs des Instituts für Grund-, Technik- und Technologiepädagogik (IFAP) und des Graduate Program in Professional and Technological Education (PROFEPT IFAP).
} 
Arbeit darauf ab, Initiativen in diesem Sinne zu fördern und so zur Popularisierung der Wissenschaft in diesem Bereich beizutragen. Zu diesem Zweck wird hier der Prozess des Aufbaus und der Verbreitung eines Audioinhalts, der auf die wissenschaftliche Verbreitung des brasilianischen EPT abzielt, über Podcast dargestellt. In diesem Sinne wird zunächst die Frage der Lücke und damit die Notwendigkeit, die Wissenschaft in diesem Bereich zu popularisieren, angesprochen. In einem zweiten Moment werden das Design und die Technologien vorgestellt, die bei der Konstruktion des EPTCastObjekts verwendet werden. Die Popularisierung der Wissenschaftlichkeit der brasilianischen beruflichen und technologischen Bildung ist das, was mit dieser Arbeit beabsichtigt ist - die nicht die einzige sein wird. Durch das, was in den vorhergehenden Abschnitten aufgedeckt wurde, soll es daher zur Popularisierung einer Wissenschaft beitragen, die manchmal noch nicht als solche verstanden wird.

Schlagworte: Podcast, Wissenschaftliche Verbreitung, Berufliche und technologische Bildung.

\section{EINLEITUNG}

Die wissenschaftliche Verbreitung ist eine Praxis, die von der Prämisse der Entfernung zwischen der wissenschaftlichen Gemeinschaft und der nicht spezialisierten Öffentlichkeit ausgeht. In diesem Sinne wurde diese kommunikative Anstrengung als pädagogisches diskursives Instrument der Wissensbildung vorgestellt, das darauf abzielt, wissenschaftliche Prozesse der breiten Öffentlichkeit näher zu bringen (MOIRAND, et ali, 2016).

Mit der Verbreitung des weltweiten Netzes von Computern über das Internet findet die wissenschaftliche Verbreitung daher mehr Raum, sich zu entwickeln. Dies wird durch die Vielfalt der digitalen Medien wahrgenommen, in denen sich diese Aktivität manifestiert: von Texten auf Blogs, Videosauf dedizierten Plattformen bis hin zu Podcasts (DE-LARA-GONZ-LEZ und DEL-CAMPO-CA-IZARES, 2018).

Mit seiner ersten Referenz aus dem Jahr 2004 ist der Medien-Podcast im Bereich der Kommunikation populär geworden. Von der zunehmenden Menge und Vielfalt der 
Optionen, normale Menschen, Unternehmen und sogar traditionelle Medienunternehmen haben ihre Inhalte über diese Medien produziert und verteilt (RIVERA SALAS e MONTOYA MÁRQUEZ, 2019).

Angesichts der bereits konsolidierten, die Podcast-Medien hat einfache und zugängliche Prozesse, ob für seine Produktion oder Verbrauch. Dies stellt sich somit als Grund dar, diese Medien bei der Verbreitung von Bildungsinhalten zu nutzen. Wenn es jedoch um die wissenschaftliche Verbreitung geht, ist diese Bewegung seltener (DANTAS-QUEIROZ, WENTZEL e QUEIROZ 2018).

Insbesondere in Bezug auf die wissenschaftliche Verbreitung in der brasilianischen beruflichen und technologischen Bildung (EPT) gibt es eine Leere. Als Instrument des sozialen Aufstiegs verstanden, hat dieser Forschungsbereich Wissen aufgebaut, das notwendig ist, um die Bedingungen der Volksarbeiterklasse zu verbessern, so dass sich in der Tat die Gesellschaft als Ganzes entwickelt (MOURA, 2013).

\section{ZIEL}

Stellen Sie den Prozess des Baus von EPTCast dar, um größere und bessere Initiativen zu fördern, um zur Popularisierung der auf dem Gebiet der brasilianischen EPT produzierten Wissenschaft beizutragen.

\section{MATERIALIEN UND METHODEN}

EPTCast wurde von denjenigen entwickelt, die die Integration von Bildung und Arbeit als weg für die soziale Entwicklung der populären Arbeiterklasse sehen, und wird durch einen Audioinhalt gebildet, der auf die wissenschaftliche Verbreitung des brasilianischen EPT abzielt. Verteilt über den Medien-Podcast, ist dies ein Bildungsprodukt, das im ersten Halbjahr 2020 gestartet und während des professionellen Master-Studiengangs Professional And Technological Education in National Network (ProfEPT) entwickelt wurde.

Die erste Herausforderung für die Konstruktion dieses Vorschlags war die Schaffung einer visuellen Identität, die die Gestaltung eines Namens, eines Slogans und eines 
grafischen Symbols erforderte. Eine visuelle Identität ist in der Regel für die bestmögliche Kommunikation durch verbale und/oder nonverbale Zeichen ausgelegt (MILLER und TOMAN, 2014). Angesichts der Notwendigkeit der Verbreitung über das Internet - die das Vorhandensein visueller Ressourcen für die Verbreitung von Inhalten stark erfordert (LIU, 2020) - war dies daher die erste Nachfrage beim Aufbau dieses Bildungsprodukts.

Dann, für die Konzeption des Namens, wurde zunächst der Vorzug für etwas Objektives gegeben, das direkt mit seiner Art der Kommunikation verbunden war. In diesem Sinne und vor allem aus dem Verständnis, dass die Namen - ob für Ideen, Produkte oder Dienstleistungen - immer in einer bestehenden Struktur aufgebaut sind, die einen neuen Namen dazu bringt, einen Teil anderer zu tragen (NDERITU PATRICK, 2016), haben wir uns entschieden, das Akronym "Professional and Technological Education" mit dem letzten Teil des Namens seiner Vertriebsmedien "cast" zu verbünden. Als Ergebnis wurde der Name EPTCast erstellt.

Dann, für die Fortsetzung der Entwicklung der visuellen Identität, wurde ein Slogan gebaut. Kurzes Wort und schnelle Assoziation mit der idee mit Namen vorgeschlagenen, ein Slogan zielt darauf ab, kurz das Konzept eines Werkes (MILLER und TOMAN, 2014). Daher haben wir uns entschieden, die Vertriebsmedien zusammen mit den vorgeschlagenen Inhalten zu beschreiben. Bald nahm der Name EPTCast den folgenden Slogan an:"Dein Podcast über professionelle und technologische Bildung".

Schließlich folgte nach der Konzeption des Namens und seinem Slogandie Konstruktion eines grafischen Symbols. Auch als Logo bekannt, ist dies ein visuelles Feature, das auf die Kommunikation mit der interessierten Öffentlichkeit abzielt. In dieser Perspektive sollte die visuelle Identität einer Marke ein einfaches und verständliches Kommunikationsinstrument sein (BOLHUIS et ali, 2015). Daher haben wir uns für den Einsatz eines Dialogballons entschieden. Schließlich haben wir für den Aufbau der aufwendigen Idee die kostenlose Version der Online-Plattform für die Erstellung von Zeichnungen und Layouts genutzt, Canva.com. 
Nach der Entwicklung der visuellen Identität hatte die Materialisierung des EPTCast Educational Product die Produktions- und Vertriebsaktivitäten. Die Produktion bestand aus der Arbeit von Scripting, Capturing und Editing Audio-Inhalte. Für diese Aufgaben wurden die Textbearbeitungssoftware Microsoft Word Version 2016, die Audiobearbeitungssoftware Audacity Version 2.3.1 und Audiofunktionen der YouTube (Audiolibrary) und Freesound.org Plattformen als arbeitsische Instrumente verwendet.

Im Gegenzug wurde die Verteilung durch die Verwendung der Anchor.fm durchgeführt. Zur Identifizierung des produzierten Materials wurden die für diese Aufgabe gewidmeten Räume direkt auf der Anchor.fm-Plattform verwendet, die automatisch den Inhalt von EPTCast in die folgenden digitalen Audioplattformen einfügte: Apple Podcasts; Breaker; Castbox; Google Podcasts; Overcast; Taschen-Güminen; RadioPublic und Spotify.

\section{ERGEBNISSE UND DISKUSSIONEN}

Die Arbeit, die während des gesamten Entwicklungsprozesses dieses Bildungsprodukts verwendet wurde, war das Ergebnis von Stunden des vorherigen Konsums von wissenschaftlichen Verbreitungsinhalten, die über Podcasts verteilt wurden. Da es jedoch im Bereich der brasilianischen PTSD im Falle dieser Besonderheiten seltene Hinweise gibt, können die folgenden Ergebnisse gleichzeitig Einschränkungen in Bezug auf ihren Aufbau und Möglichkeiten für die Entwicklung robusterer Vorschläge aufzeigen.

\section{VISUELLE IDENTITÄT}

Durch die Nutzung der kostenlosen Version der Canva.com Plattform (https:canva.com) begann der Aufbau der visuellen Identität des EPTCast Educational Product. Für Ihre Leinwand wurde die Größe von 1500 x 380 Pixel und Farbe \#000000 gewählt. Für den Namen, die Montserrat Classic typografische Schrift artografische Schrift in fett stilisiert Stil mit Größe 50 und Farbe der \#000000. Dann wurde der Slogan bearbeitet,der die gleiche typografische Schrift artundiert und Stil auf den Namen angewendet, aber erhielt Änderungen in Größe und Farbe, bzw. 38 und \#ffffff. 
Schließlich wurde in der Option "Formen" unter "Elemente" auf der in Gebrauch befindlichen Plattform eine Dialog-Ballon ausgewählt. Nach dem Einfügen wurde die Größe des Elements auf Größe 365 um 290 Pixel und Farbe \#ffbf00 (Abbildung 1) geändert.

Abbildung 1: Visuelle Identität des EPTCast-Bildungsprodukts.

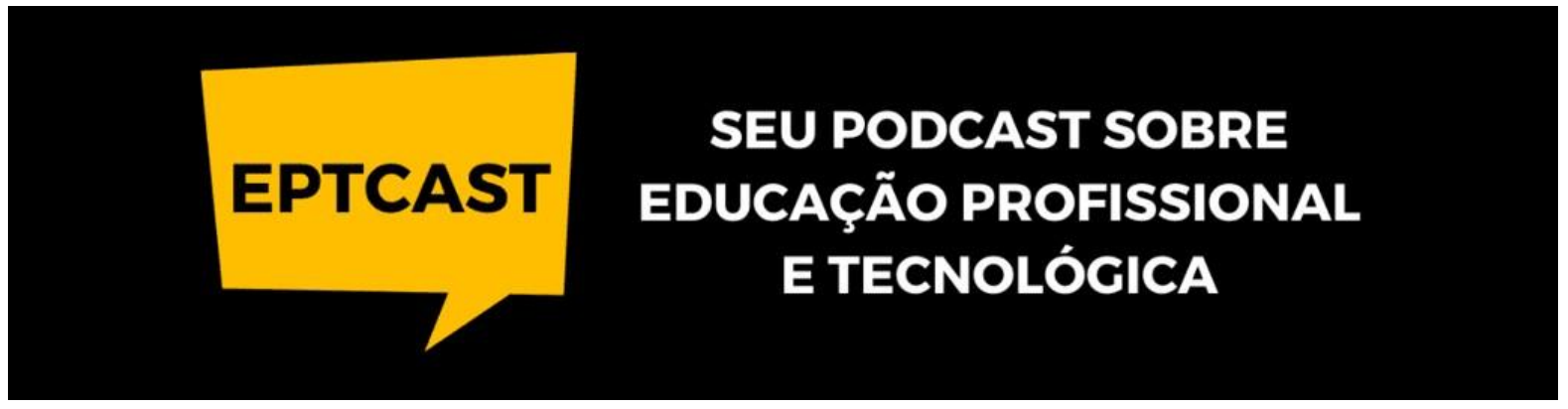

Die visuelle Identität dieses Bildungsprodukts wurde so konzipiert, dass es in Zukunft in digitalen Räumen und physischen Werbeprodukten angewendet werden kann. Daher wurde seit Beginn seiner Konstruktion festgestellt, dass diese Identität durch ein einfaches Bild und eine mehrfache Anwendbarkeit charakterisiert werden sollte.

Im Rahmen dieses Vorschlags wurden auf den Namen Spezifikationen angewendet, die den geplanten Entsprechen. Um das zentrale Merkmal des Namens zu erhalten, bewahrte der Slogan die gleiche Idee, jedoch, angesichts der größeren Anzahl von Buchstaben, wurde seine Größe reduziert und, um vor der Leinwand gelesen zu werden, wurde seine Farbe geändert. Der Dialogballon wurde für die Anerkennung des kommunikativen Vorschlags beantragt. Seine differenzierte Farbe und die Möglichkeit, den Namen davor zu positionieren, wurde so konzipiert, dass sie auch unabhängig vom Slogan präsentiert werden konnten.

\section{PRODUKTION}

Von Januar 2020 bis September desselben Jahres führte die Produktion dieses Bildungsprodukts zu 19 Audios (\#00 bis \#18). Zunächst - bis zur Veröffentlichung des 12. - wurde der Inhalt durch wiederkehrende Themen in der Literatur der brasilianischen PtSD entwickelt. Ab dem 14. begann es, durch Vorschläge von 
Themen, die vom Publikum gesendet wurden, zu produzieren und zu veröffentlichen, das die Kommunikationskanäle eptcast.com.br (Kommentare) und die E-Mailcontato.eptcast.com.br. Der 13. Audio-Posted sollte die bisher präsentierten Inhalte zusammenfassen und die Zuschauerbeteiligung anregen.

\section{SCRIPTING}

Nach Abschluss der visuellen Identität wurde das Skripting mit der proprietären Textbearbeitungs software Microsoft Word, Version 2016 (https://office.com) durchgeführt. Aus der Verwendung dieses Werkzeugs begann die Konstruktion der in 03 Teilen entwickelten Inhalte, "Präsentation", "Evolution" und "Schlussfolgerung" (Tabelle 1).

Die "Präsentation" wurde in andere 03-Teile unterteilt, wo die erste "Eröffnung" der Begrüßung, Präsentation des Ansagers und der Identifizierung des Programms gewidmet war. Dann konzentrierte sich "Kontext" darauf, die Konjunktur des angesprochenen Phänomens zu identifizieren. Zum Abschluss des Blocks "Präsentation" wird der Block "Call to action" verwendet, zu dem das Publikum am Ende des Audios auf elektronischem Wege zur Interaktion eingeladen wird.

Der nächste Moment, "Evolution", wurde in 04 Teile unterteilt. Das erste, "Phänomen", war der Zusammenfassung über das behandelte Objekt gewidmet. Als nächstes waren "Vorläufer" und "Merkmale" Räume, die sich auf den Ansatz des Entwicklungsprozesses des betreffenden Phänomens und seiner Eigenschaften konzentrierten. Schließlich widmete sich "Desdobramentos" den Folgen im sozialen Kontext, insbesondere für Bildung und Arbeit.

Die "Schlussfolgerung" wurde in 03 Teile unterteilt. Die erste, "Reflexion", war den prägnanten Bemerkungen zu diesem Thema gewidmet. Im nächsten Moment, "Referenzen", zielte darauf ab, das Publikum über den Ort der Unterbringung des Materials, das bei der Konstruktion des Skripts verwendet wird, zu informieren. Schließlich wurde die Funktion "Call to Action" verwendet. 
Tabelle 1 - Erzählschema des Skripts.

\begin{tabular}{|c|c|c|}
\hline Apresentação & Evolução & Conclusão \\
\hline Abertura & Fenômeno & Reflexão \\
\hline (30 segundos) & (01 min. e 30 segs.) & (02 mins. e 30 segs.) \\
\hline Contexto & Antecedentes & Referências \\
\hline (01 minuto) & (01 min. e 30 segs.) & (30 segundos) \\
\hline \multirow{3}{*}{$\begin{array}{r}\text { Call to action } \\
\text { (30 segundos) }\end{array}$} & Características & Call to action \\
\hline & (03 minutos.) & (01 minuto) \\
\hline & $\begin{array}{c}\text { Desdobramentos } \\
(03 \text { minutos. })\end{array}$ & \\
\hline 02 minutos & 09 minutos & 04 minutos \\
\hline
\end{tabular}

\section{5 minutos}

Die Kompositionselemente des Erzählschemas wurden so gedacht, dass der Hörer die Beziehung zwischen dem behandelten Phänomen und der aktuellen Realität im Kontext von Bildung und Arbeit verstehen sollte. Die Zeit, die jeder Abteilung und ihren Unterteilungen gewidmet war, wurde so festgelegt, dass sie eine Standardisierung und damit eine bessere Organisation für den Bau zukünftiger Routen ermöglichten.

Von den insgesamt 18 produzierten Audiodateien entsprachen die Nummern \#00, \#04, \#08, \#10 und \#12 aufgrund der Art ihrer Vorschläge nicht der oben genannten Regelung. Und von der \#13, wegen der Pluralität unter den Vorschlägen der erhaltenen Themen, erfuhr das Erzählschema einige Änderungen in der Sektion "Evolution". Der Kern dessen, was in dem oben vorgestellten Schema gebaut wurde, blieb jedoch erhalten, bis das letzte Thema.

Tabelle 2 zeigt die Anzahl der angesprochenen Audios und Themen. 
Tabelle 2 - Themen, die an jedes Audio adressiert sind.

\begin{tabular}{c|c}
\hline Número & Assunto \\
\hline$\# 00$ & O que é o EPTCast?"; \\
\hline$\# 01$ & Manual ou Intelectual? \\
\hline$\# 02$ & Onde estão as fontes? \\
\hline$\# 03$ & Natural ou Social? \\
\hline$\# 04$ & O que é... Ciência? \\
\hline$\# 05$ & A crise de 29 \\
\hline$\# 06$ & Globalização \\
\hline$\# 07$ & Matemática \\
\hline$\# 08$ & A Fala Docente! \\
\hline$\# 09$ & O que é Materialismo Histórico Dialético? \\
\hline$\# 10$ & Final de Temporada \\
\hline$\# 11$ & Percurso Histórico Estrutural da EP Brasileira \\
\hline$\# 12$ & A Cientificidade da Música \\
\hline$\# 13$ & Educação Profissional e Propedêutica \\
\hline$\# 14$ & Redação \\
\hline$\# 15$ & Cursos Técnicos \\
\hline$\# 16$ & Formação de Professores para EPT \\
\hline$\# 17$ & Novas Exigências e Velhas Transformações \\
\hline$\# 18$ & \\
\hline
\end{tabular}

Bis zum Audio-\#16 Technischen Kursen betrug die Zeit jeder Produktion 15 Minuten.

Daraus \#17 also neue Anforderungen und alte Transformationen; und \#18 Lehrerausbildung für EPT, wurden mit einer Gesamtzeit von 12 Minuten produziert und 
veröffentlicht. Diese Änderung wurde aufgrund von Anregungen aus dem Publikum gefördert.

\section{ERFASSEN}

Nach dem Abschluss des Erzählschemas und der Ausarbeitung des ersten Drehbuchs begann die Audioproduktion. Zu diesem Zweck wurde die Aufnahme durch AudacitySoftware, Version 2.3.1 (https://audacityteam.org) auf einem tragbaren Mikrocomputer-Modell Lenovo IdeaPad 310 und mit Hilfe eines generischen Kondensatormikrofons, dem BM-800 (Abbildung 2) installiert durchgeführt.

Abbildung 2: Audioaufnahme.

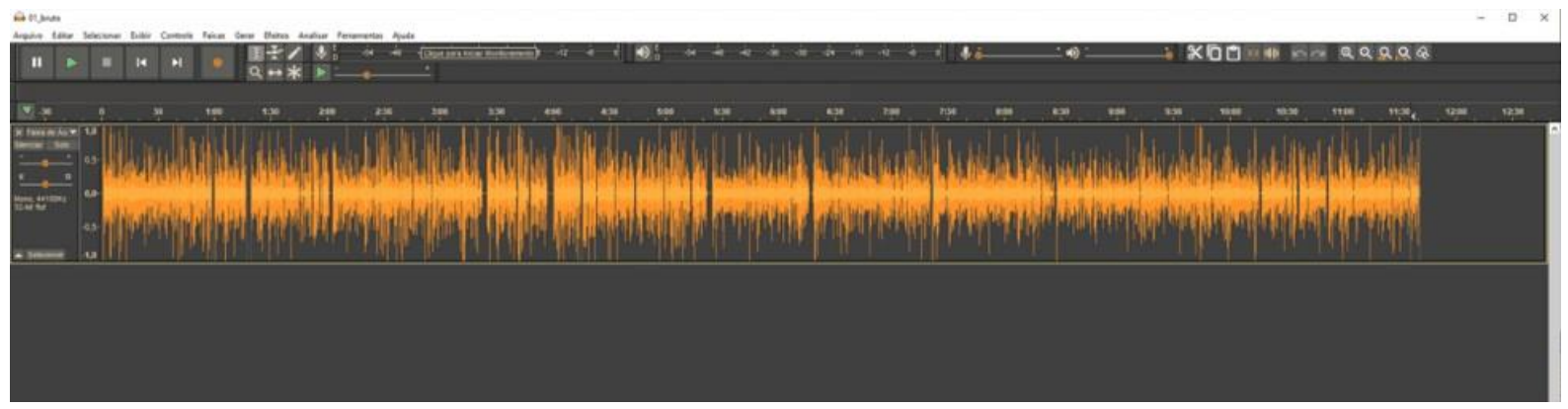

Die Audioaufnahme stellte den Beginn des Prozesses der Materialisierung des geplanten Ziels dar. Aufgrund der mangelnden Erfahrung in dem, was sich auf die Erzählung von schriftlichen Inhalten für die Audioverteilung bezog, war die Aufnahmegröße/-lautstärke manchmal mehr als doppelt so lang wie für jeden Ton festgelegt. Die obige Abbildung zeigt den aufgezeichneten Inhalt ohne fehlerbehaftete Erfassungsprozesse.

\section{EDITION}

Nach der Aufnahme des Audios und dem Entfernen des Überschusses wurde der Bearbeitungsprozess eingeleitet. Für diese Phase wurden die Audioressourcen der YouTube-Plattformen (https://youtube.com/audiolibrary) zum Einfügen von Musiktiteln und Freesound.org (https://freesound.org) für das Einfügen von Soundeffekten 
verwendet. Beide Plattformen verfügen über Inhalte ohne finanzielle Kosten (Abbildung 3).

Abbildung 3 - Audiobearbeitung.

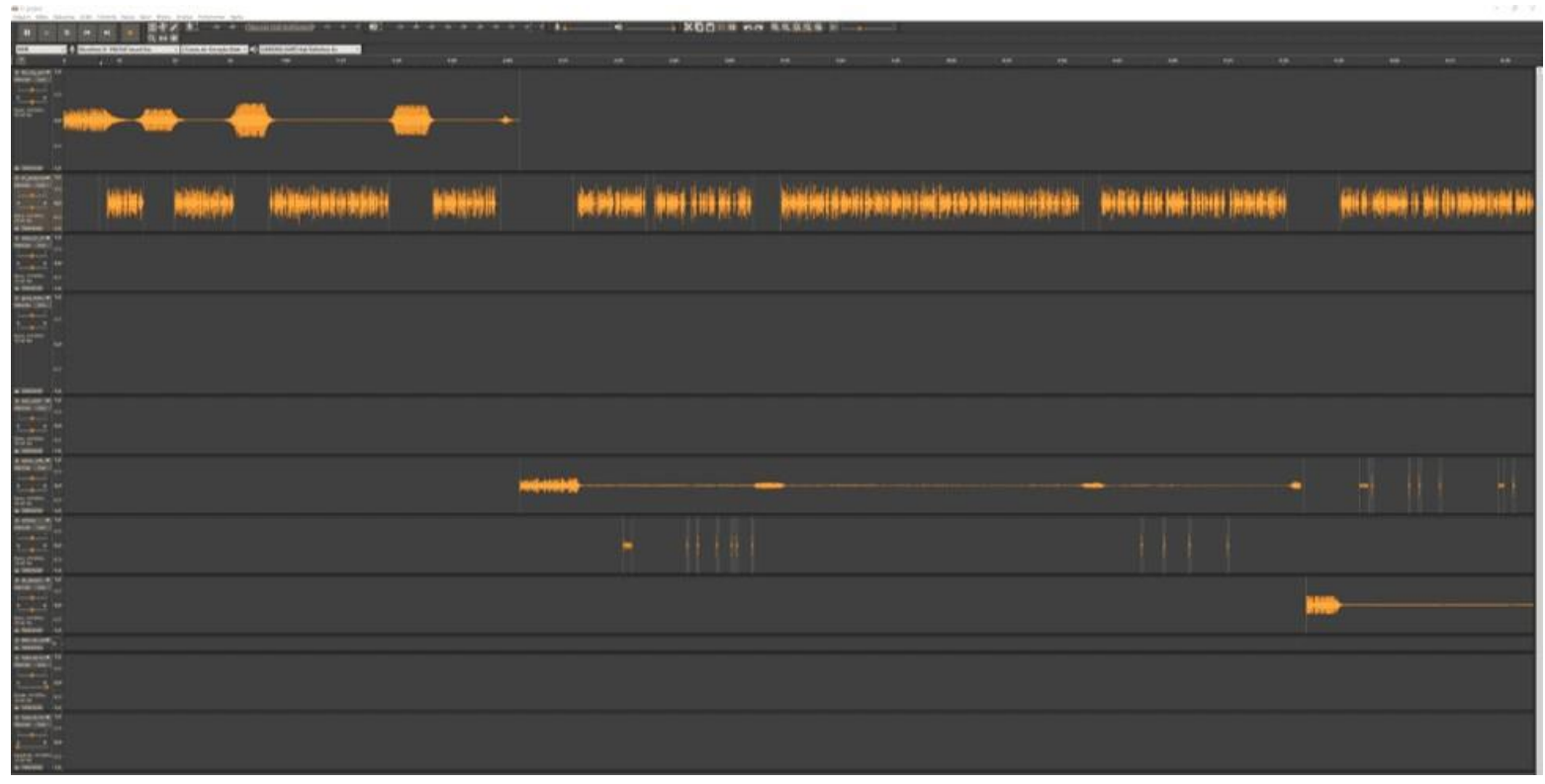

Die Möglichkeit, Musiktitel und Soundeffekte einzufügen, war das Ergebnis der Erfahrung des vorherigen Konsums von Inhalten, die über Podcasts verteilt wurden. Während des gesamten Bearbeitungsprozesses wurde auch wahrgenommen, dass diese Ressource, auf einer gewissen Ebene, sich als in der Lage präsentierte, das Vorhandensein bestimmter unerwünschter Einfügungen zu mildern, die von der Audioaufnahme in der häuslichen Umgebung stammen.

Um die Rede des Erzählers mit den ursprünglichen Argumenten der Forschungsquellen zu differenzieren, wurde der Inhalt von 04 Studenten des Integrierten Gymnasiums der Bundesanstalt für Bildung, 02 des Campus Von Amapá, Santana, campus Santana; und 02 von Rio Grande do Sul, Campus Osório. In diesem Sinne waren die eingeladenen Schüler dafür verantwortlich, die direkten Zitate zu erzählen, die in den Text des Skripts eingefügt wurden. 


\section{VERTEILUNG}

Unter den vielen Optionen für die Distribution von EPTCast haben wir uns für die Anchor.fm - http://anchor.fm entschieden. Dies war eine Entscheidung, die auf zwei Grundlagen beruhte: Totale Trinkgeld in seiner Handhabung; und automatische Verteilung von Inhalten, die auf anderen Plattformen veröffentlicht werden.

\section{IDENTIFIKATION DES MATERIALS}

Durch Senden der Inhalte, die über die für diese Aufgabe vorgesehene Ressource erzeugt wurden (Click to upload or drag files here),wurde das Audio hochgeladen, sodass die Wiedergabe ermöglicht wurde, sodass die Qualität des zu verteilenden Inhalts überprüft werden konnte (Abbildung 4).

Abbildung 4: Einfügen des Audios und Identifizierung des Materials.

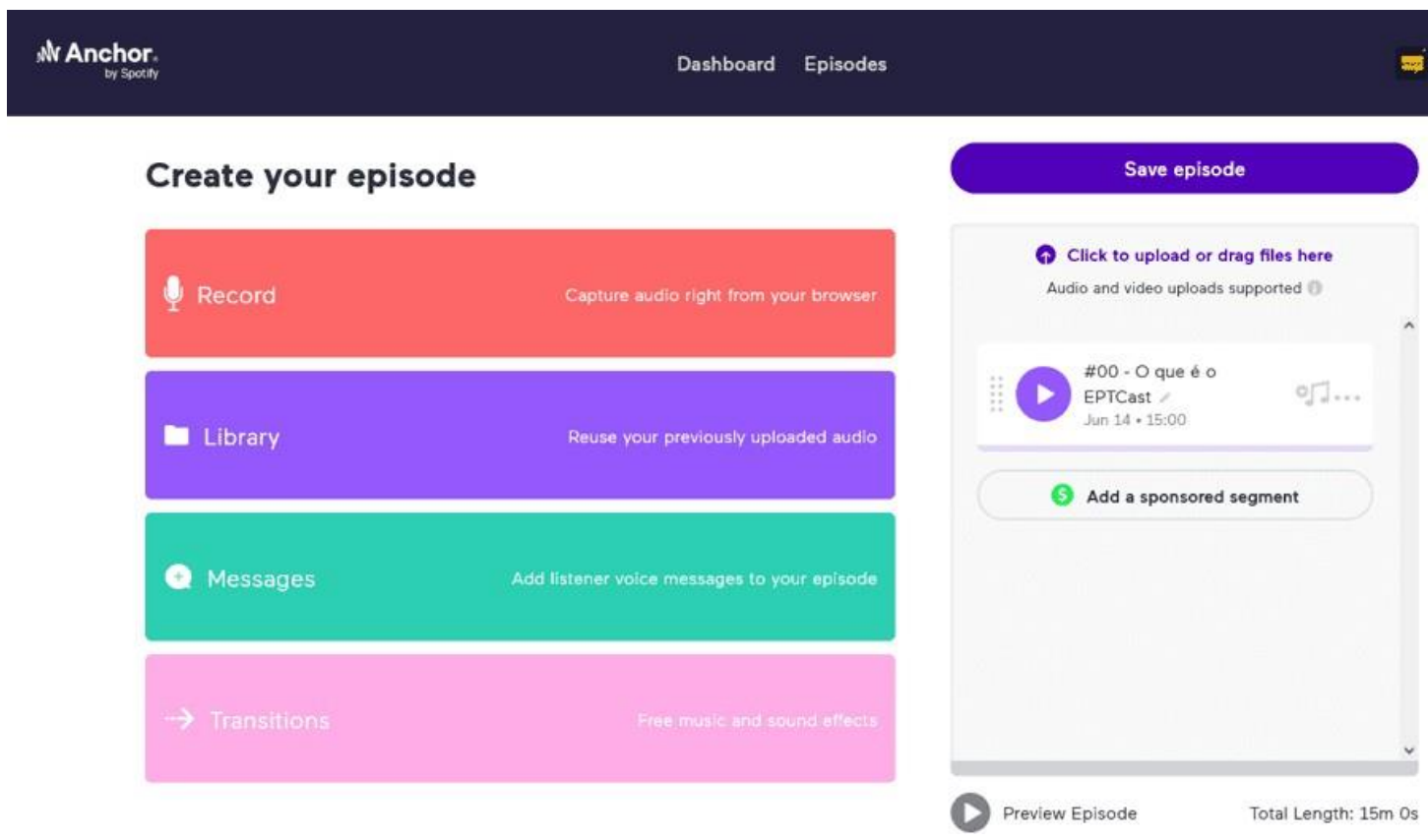

Nach dem Einfügen des Audios forderte die Plattform grundlegende Informationen zur Identifizierung des Inhalts an. Diese waren: Titel (Episode title), Beschreibung (Episode description), Staffel (Season number), Inhaltsnummer (Episode number), 
Inhaltstyp zwischen: komplett, Trailer oder Bonus (Full; Trailer; Bonus) und schließlich, ob dies ist nutzerfreundlicher Inhalt oder nicht (Clean; Explicit) (Abbildung 5).

Abbildung 5 - Kennung des Materials.

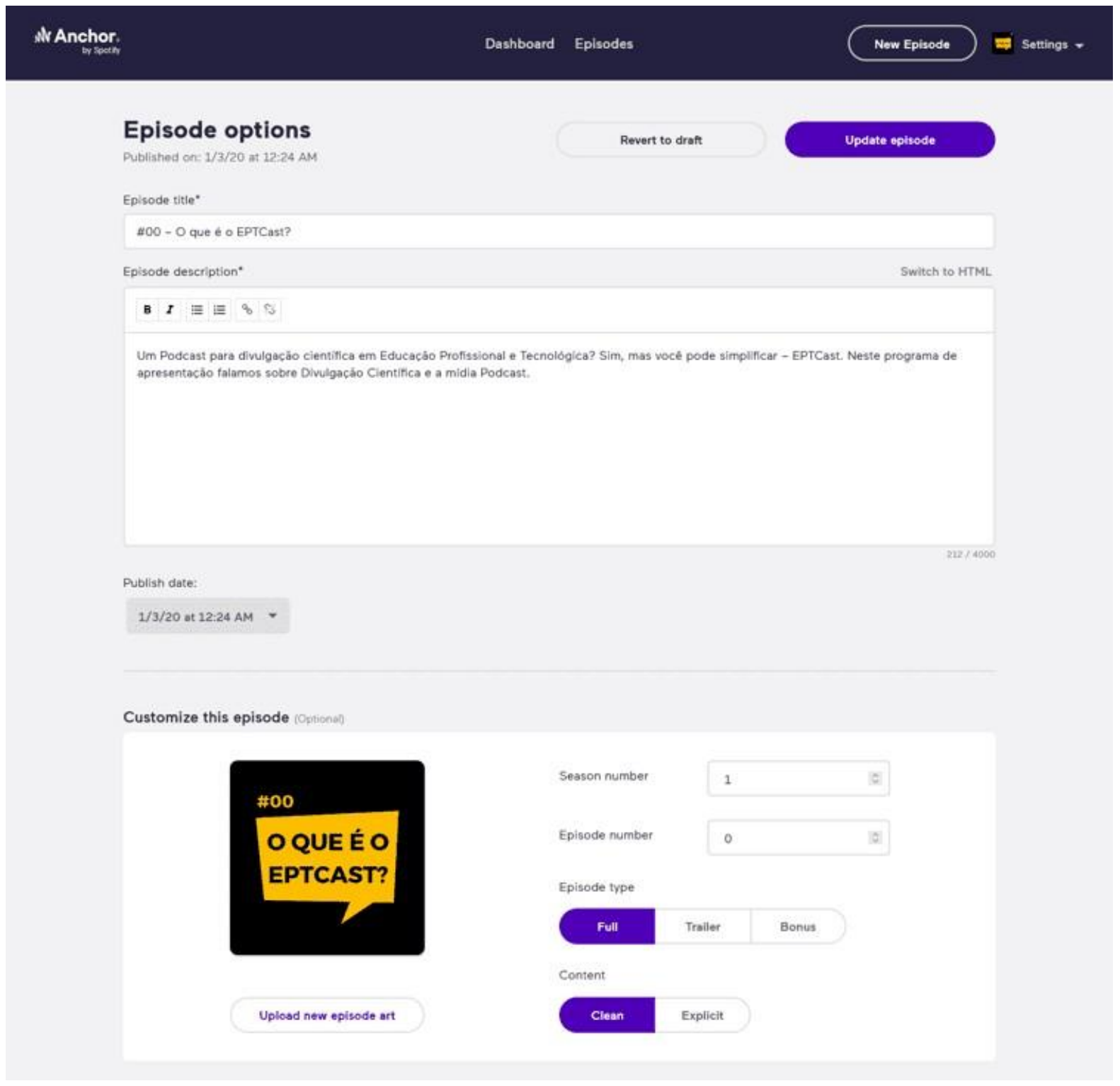

Nach dem Einfügen der zur Identifizierung des Inhalts angeforderten Informationen wurde das Audio auf dieser Plattform und auch in der Musikstreaming-Plattform Spotify (https://spotify.com) zur Verfügung gestellt, so dass sein Verbrauch in den ersten Momenten möglich war. 


\section{DIGITALE AUDIOPLATTFORMEN}

Trotz der Automatisierung des Verteilungsprozesses auf anderen Plattformen hat Anchor.fm diese Aufgabe nicht gleichzeitig auf allen Plattformen ausgeführt, mit der sie verbunden ist. Jedoch, als das Senden von Audio erhöht EPTCast Online-Inhalte, wurde festgestellt, dass die Zugriffsoptionen wuchsen. Bei der Veröffentlichung der \#07 Globalisierung wurde festgestellt, dass die Verteilung inre maximale Anzahl von Plattformen erreichte, 09 (Tabelle 3).

Tabelle 3 - Zugangsplattformen.

\begin{tabular}{c|c}
\hline NOME & ENDEREÇO (URL) \\
\hline Anchor & anchor.fm/eptcast \\
\hline Breaker & breaker.audio/eptcast \\
\hline Castbox & castbox.fm/channel/id2581365 \\
\hline Google Podcasts & google.com/podcasts?feed=aHROcHM6Ly9hbmNob3luZm0vc \\
\hline Apple Podcasts & podcasts.apple.com/us/podcast/eptcast/id1494934631 \\
\hline Overcast & overcast.fm/itunes1494934631/eptcast \\
\hline Pocket Casts & pca.st/8znyqbx5 \\
\hline RadioPublic & radiopublic.com/eptcast-WkaRNN \\
\hline Spotify & open.spotify.com/show/1hZ3KSkDDZOHuLmIPD6dh8
\end{tabular}

Die Verteilung über die ausgewählte Plattform präsentierte sich als ein Erlebnis des einfachen Verständnisses und auch der einfachen Handhabung. Die kostenlose Einfügung in andere digitale Plattformen stellte sich als wichtiges Gefälle dar, da sich diese Aktivität als Zeitersparnis erwies, eine Ressource, die bei der Erfüllung anderer Aufgaben dieses Bildungsprodukts eingesetzt wurde.

\section{ENDGÜLTIGE ÜBERLEGUNGEN}

Die Popularisierung der Wissenschaftlichkeit der brasilianischen beruflichen und technologischen Bildung ist das, was mit dieser Arbeit beabsichtigt wurde - die nicht die einzige sein wird. Durch das, was in den vorhergehenden Abschnitten aufgedeckt 
wurde, soll es daher zur Popularisierung einer Wissenschaft beitragen, die manchmal noch nicht als solche verstanden wird.

Die gesellschaftliche Entwicklung der volkstümischen Arbeiterklasse durch die Entwicklung einer wissenschaftlichen Kultur ist unsere Absicht. In diesem Sinne nutzen wir ein dezentralisiertes Medium, um diese Themen zu popularisieren, die, obwohl sie sich auf die Emanzipation derjenigen konzentrieren, die bisher nur innerhalb des reservierten Zyklus gefunden und gehört werden müssen.

Von hier aus hoffen wir auf größere und bessere Initiativen, die darauf abzielen, diese Themen zu popularisieren.

\section{VERWEISE}

MOIRAND, S. et ali. La vulgarisation scientifique au croisement de nouvelles sphères d'activité langagière. Bakhtiniana, Rev. Estud. Discurso, vol. 11 n. 2, p. 137-161, 2016.

DE-LARA-GONZÁLEZ, A.; DEL-CAMPO-CAÑIZARES, E. El podcast como medio de divulgación científica y su capacidad para conectar con la audiencia. Revista Mediterránea de Comunicación, vol. 09 n. 1 p. 347-359, 2018.

RIVERA SALAS, P.E.; MONTOYA MÁRQUEZ, O. R. Evaluación de los podcasts de noticias: un acercamiento a emisoras de la ciudad de puebla. Revista de Comunicación de la SEECI. n. 48, p. 109-123, 2019.

DANTAS-QUEIROZ, M. V.; WENTZEL, L. C. P.; QUEIROZ, L. L. Science communication podcasting in Brazil: the potential and challenges depicted by two podcasts. An. Acad. Bras. Cienc., v. 90, n. 2, p. 1891-1901, 2018.

MOURA, D. H. Ensino médio integrado: subsunção aos interesses do capital ou travessia para a formação humana integral? Educ. Pesqui., v. 39, n. 3, p. 705-720, 2013. 
NDERITU PATRICK G. Bilingual naming of business premises, products and services in Murang'a county. Relatório de Projeto de Pesquisa (Mestre em Linguística) Universidade de Nairóbi, 2016.

LIU, X. Application of the Internet Technology in the Visual Communication Design. In: Atiquzzaman M., Yen N., Xu Z. (eds) BDCPS 2019, AISC 1117, p. 1701-1706, 2020.

MILLER, D. W.; TOMAN, M. An analysis of rhetorical figures and other linguistic devices in corporation brand slogans. Journal of Marketing Communications, v. 22, n. 5, p. 474-493, 2014.

Eingereicht: Juni 2021.

Genehmigt: Juni 2021. 